Wild-type genotypes were found in $34 \%$ of patients (group $A$ ), whereas the remainder were $I L-1 R N 2$ carriers with or without IL-1B polymorphisms (36\%; group B) or had at least one IL-1B polymorphism and wild-type IL-1RN (30\%; group C). Although chemotherapy response rates were similar in the three groups, progression-free survival and overall survival were significantly better in groups $A$ and $B$ than in group C. This suggests that the combination of $I L-1 B$ polymorphisms and wild-type $I L-1 R N$ has an adverse effect on prognosis in this setting.

The authors also discuss the potential role of recombinant human IL-1RN in the treatment of metastatic cancer, and recommend that patients should be stratified according to IL-1B/IL-1RN genotype.

Original article Graziano F et al. (2005) Prognostic role of interleukin- $1 \beta$ gene and interleukin - 1 receptor antagonist gene polymorphisms in patients with advanced gastric cancer. JClin Oncol 23: 2339-2345

\section{Mitochondrial mutations and colorectal cancer prognosis}

Human mitochondrial DNA ( $m$ tDNA) is thought to be more prone to mutations than nuclear DNA, and mutations within this 16,569 basepair genome have been reported in different types of cancers. Lièvre and colleagues have now identified a mutational 'hotspot' within the non-coding, displacement-loop (D-loop) region of the mitochondrial genome; their recent paper relates these findings to the clinicopathologic characteristics of a series of patients with colorectal cancer.
To search for mutational hotspots, the investigators amplified and sequenced the entire mitochondrial genome using DNA isolated from 11 colorectal tumors. In order to differentiate between germline and somatic mutations, matched healthy tissue - taken from the surgical margins-was also analyzed. A total of 10 somatic mutations were found in 7 of the tumor samples, and 8 of these were in the D-loop region of the mtDNA. Specifically, the mutations occurred within the D310 sequence of the D-loop region in six (54\%) of the patients; the authors therefore considered this region to be a hotspot of mtDNA mutations in patients with colorectal cancer.

The team went on to amplify and sequence the D-loop region of the mtDNA in an additional 365 patients who underwent colorectal cancer resection. A somatic D-loop mutation was found in 142 (39\%) of the tumors, and the D310 sequence was affected in 132 cases (36\%). Three-year survival was significantly lower in patients with D-loop mutation than in those without ( $53.5 \%$ vs $62.1 \%, P=0.05)$. This corresponded to a $40 \%$ increase in the risk of death, after adjusting for factors such as age and disease stage. In addition, the presence of a D-loop mutation was associated with resistance to adjuvant chemotherapy in patients with stage III colon cancers.

In summary, the $D$-loop region of the human mitochondrial genome appears to be a hotspot for somatic mutations in colorectal cancer, and mutations in this region are linked to poor clinical outcomes.

Original article Lièvre A et al. (2005) Clinical value of mitochondrial mutations in colorectal cancer. J Clin Oncol 23: $3517-3525$ 